\title{
Efecto de un aceite de trementina obtenido de Pinus tropicalis Morelet sobre la biología de una cepa de Aedes (Stegomyia) aegypti Linnaeus, 1762 resistente a insecticidas
}

\author{
Maureen Leyva ${ }^{1}$, María del Carmen Marquetti ${ }^{1}$, Leidys French ${ }^{1}$, Domingo Montada ${ }^{1}$, Olinka Tiomno ${ }^{2}$, Juan E. \\ Tacoronte ${ }^{3}$ \\ 1 Instituto Medicina Tropical "Pedro Kouri" (IPK). Autopista Novia del Mediodía Km 6 1⁄2, Apartado 601, La Lisa, Ciudad de La \\ Habana, Cuba. \\ 2 Centro de Investigaciones Químicas (CIQ). Infanta y Palatino. Plaza de la Revolución, Ciudad de la Habana, Cuba. \\ 3 Proyecto Prometeo, SENESCYT, Universidad Central del Ecuador, Facultad de Ingeniería Química, Quito, Ecuador
}

\begin{abstract}
Resumen
Correspondencia

M. Leyva Silva

E-mail: maureen@ipk. sld. cu

FAX: 53-7-2046051

Recibido: 22 marzo 2013

Aceptado: 20 septiembre 2013

Publicado on-line: 22 octubre 2013

En este estudio se determinó la influencia de la $\mathrm{CL}_{95}$ del aceite de trementina sobre la biología de una cepa cubana de Aedes aegypti colectada en la localidad de San Miguel del Padrón, La Habana, Cuba. La captura se realizó en el 2011, durante una etapa intensiva de control. Se determinó el estado de susceptibilidad a insecticidas de la cepa en estudio y se expuso a una $\mathrm{CL}_{95}$ de un aceite de trementina modificado por fotoisomerización y obtenido de Pinus tropicalis. Se obtuvo alta mortalidad larval y pupal en las larvas sobrevivientes de la exposición. Para la variante I se obtuvo un $70 \%$ de inhibición y para la variante II un $68 \%$. Las hembras sobrevivientes de ambas variantes disminuyeron su fecundidad con respecto al control. No hubo afectación de la fertilidad.
\end{abstract}

Palabras clave: Trementina, Inhibición del desarrollo, Culicidae, Cuba

\begin{abstract}
Effect of a turpentine oil obtained from Pinus tropicalis Morelet on the biology of a strain Aedes (Stegomyia) aegypti Linnaeus, 1762 insecticide resistant

This study investigated the influence of turpentine oil CL95 on the biology of a Cuban strain of Aedes aegypti collected in the town of San Miguel del Padrón, Havana, Cuba. The collect was in 2011 during an intensive phase control. We determined the insecticide susceptibility status of the strain under study and exposed to an LC95 of a modified Pinus tropicalis turpentine oil obtained by photoisomerization. High mortality was obtained in larval and pupal larvae surviving exposure. For I variant was obtained a $70 \%$ inhibition and for the II variant $68 \%$. Surviving females from both variants decreased their fertility regarding control. No impaired fertility
\end{abstract}

Key words: Turpentine, Growth inhibition, Culicidae, Cuba

\section{Introducción}

Desde su descubrimiento, los plaguicidas químicos han sido el método más empleado para el control de mosquitos transmisores de enfermedades (Bourguet et al. 2000, Bouvier et al. 2001). La aplicación de insecticidas en la fase adulta, tanto en tratamientos intra como en extradomiciliarios se utiliza para obtener una reducción rápida de las poblaciones en los casos de epidemia trasmitidas por vectores (Bisset 2002). Desafortunadamente, dicha reducción suele ser transitoria si no se apli- 
can correctamente los plaguicidas. Está bien establecido que la resistencia a los mismos puede desarrollarse rápido en algunas poblaciones y lentamente en otras y su desarrollo depende de factores intrínsecos a la especie y operacionales. Estos últimos referidos al manejo y aplicación del programa de control, y principalmente a la frecuencia y dosis en las aplicaciones de insecticida (Keiding 1986; Bisset 2002, Montilla et al. 2007, Rodríguez et al. 2009, 2010, Llinas et al. 2010)

Numerosos productos químicos que son producidos en forma de metabolitos secundarios por las plantas pueden actuar como insecticidas (Shallan et al. 2005). Muchos países se enfocan en la necesidad de realizar investigaciones con productos naturales, con el fin de encontrar nuevos métodos de control alternativos que reduzcan la incidencia de enfermedades trasmitidas por vectores y la aplicación de plaguicidas sintéticos (Dharmagadda et al. 2005). Las plantas, con su gran diversidad estructural, proporcionan una importante fuente para el control de plagas (Cantin et al. 2001) por su efectividad, su rápida biodegradación y sus pocos efectos adversos al medio ambiente (Conti et al 2010). El estudio de sus propiedades incluyen la repelencia (Garcia et al. 2005, Leyva et al. 2012), inhibición de la alimentación (Erturk et al. 2006), de la oviposición (Pascual-Villalobos et al. 2003) y del crecimiento y desarrollo en insectos (Pascual-Villalobos et al. 1998, Barra et al. 2007).

En estudios anteriores, realizados en nuestro laboratorio con el aceite esencial de Pinus tropicalis, (Morelet, 1851), se obtuvo actividad larvicida y ovicida en larvas de Aedes (Stegomyia) aegypti Linnaeus, 1762 (cepa Rockefeller) susceptibles a insecticidas (Leyva et al. 2009). En vista de estudiar los mejores candidatos naturales para el control de mosquitos, se evaluó la capacidad larvicida e inhibitoria del desarrollo de aceites de trementina sobre la misma cepa (Leyva et al. 2010). El aceite de trementina es el término aplicado a la oleorresina de color pardo, que se obtienen por destilación de la resina de pino en Asia, Europa y América. Cuando es sometida a un proceso de destilación, produce la esencia de trementina dejando como residuo la colofonia. Es un líquido incoloro, tiene una densidad relativa de 0,86 a $0,88 \mathrm{~g} / \mathrm{cm}^{3}$ y un punto de ebullición de $155^{\circ} \mathrm{C}$. Está compuesto de una mezcla de terpenos ( $\alpha$-pineno, $\beta$-pineno y canfeno). En estado líquido, es insoluble en agua, ligeramente soluble en alcohol diluido, e insoluble en éter (Kirk 1983).

El objetivo de este estudio fue determinar, en una cepa de Aedes aegypti colectada en terreno, el efecto producido en las larvas sobrevivientes de la exposición a una dosis subletal de aceite de trementina modificado.

\section{Materiales y Métodos}

\section{Determinación del estado de resistencia y lo susceptibilidad a insecticidas de la cepa colectada}

La cepa de Ae. aegypti, utilizada en este estudio, fue colectada en el municipio San Miguel del Padrón, situado en La Habana, Cuba, en el año 2011, durante una etapa intensiva de control vectorial. La cepa se capturó en los estadios larva y pupa. Siguiendo la metodología del Manual de Indicaciones Técnicas del Insectario (Pérez et al. 2004), se estabilizó en el insectario del departamento de Control de Vectores de Instituto de Medicina Tropical "Pedro Kouri", Cuba, hasta la F3, generación utilizada en este estudio.

Para determinar el estado de resistencia y /o susceptibilidad a insecticidas en la fase adulta se utilizaron papeles impregnados con Lambadacialotrina $0.05 \%$, Deltametrina 0. 05\%, Malatión 5\% y Bendiocarb 0. 1\% suministrados por la Organización Mundial de la Salud. Para la realización de los bioensayos se conformaron grupos de 20 mosquitos hembras de 3 días de edad, sin alimentar, 1 control y 4 réplicas. Se expusieron por un periodo de 1 hora según metodología WHO 1981 y a su vez estos bioensayos se replicaron 3 veces. Al final de los períodos de exposición, los mosquitos adultos se transfirieron a tubos de reposo y mantenidos en posición vertical por 24 horas. Para la lectura de la mortalidad se utilizó el criterio de la OMS $1981 \mathrm{WHO/CDC/CPC/MAL.} \mathrm{98.} 12$ página 17: $99-100 \%$ de mortalidad indica susceptibilidad; mortalidad entre $80-97 \%$ indica la posibilidad de una resistencia que necesita ser verificada; y menor de $80 \%$ indica resistencia. Para la determinación del $\mathrm{TL}_{50}$, se conformaron grupos de 20 mosquitos hembras de 3 días de edad, sin alimentar, que fueron expuestas a diferentes tiempos, según metodología WHO 1981. Utilizando 1 control y 4 réplicas. La temperatura y la humedad relativa fueron registradas durante los periodos de exposición y descanso. 
Por otra parte, se evaluó la susceptibilidad y/o resistencia al insecticida organofosforado temefos, a través de los bioensayos de susceptibilidad para larvas (OMS, 1981). Se utilizaron larvas de tercer estadío tardío o cuarto temprano de Ae. aegypti. Se aplicaron cinco o más concentraciones del insecticida con acetona como diluente. Se emplearon cuatro réplicas y un control por cada concentración que causaron mortalidades entre 2 y $98 \%$. La mortalidad se determinó 24 horas después de aplicado el insecticida.

Todos los resultados se analizaron mediante el programa Probit-logaritmo de Raymond (1985). Con la $\mathrm{CL}_{50}$ se calculó el Factor de Resistencia $\left(\mathrm{FR}_{50}\right)$, comparando el valor de la cepa problema con el de la cepa susceptible Rockefeller:

$$
F R_{50}=\frac{C L_{50} \text { cepa problema }}{C L_{50} \text { cepa Rockefeller }}
$$

Se utilizó como criterio de susceptibilidad las siguientes relaciones:

Si $\mathrm{FR}_{50} \leq 5$ : Susceptible.

$5<\mathrm{FR}_{50}<10$ : Resistencia moderada.

$\mathrm{FR}_{50}>10$ : Resistente.

\section{Obtención del aceite de trementina modificado}

El aceite de trementina modificado se obtuvo por tratamiento del aceite de trementina, recién destilado de la oleorresina de Pinus tropicalis, en condiciones catalíticas heterógeneas ácidas (pentóxido de divanadio, $\mathrm{V}_{2} \mathrm{O}_{5}$, pentóxido de diniobio, $\mathrm{Nb}_{2} \mathrm{O}_{5}$, montomorillonita y arcillas pilareadas con sales de $\mathrm{Cr}^{+6} \mathrm{y} \mathrm{Mo}^{+7}$ ), bajo agitación (> $600 \mathrm{rpm}$ ) $y$ en presencia de luz solar ambiental durante 2-3 horas. El producto de reacción fue sometido a un proceso de separación-purificación vía cromatografía columnar $\left(\mathrm{SiO}_{2} /\right.$ difenilamina como fase sólida y diclorometano-acetato de etilo 30: $70 \mathrm{v} / \mathrm{v}$ como eluyente) y caracterizado mediante técnicas espectrométricas (FTIR, RMN) y CG.

\section{Evaluación de la actividad inhibidora del aceite de trementina (modificado por foto- isomerización) sobre larvas de Aedes aegypti de la cepa San Miguel del Padrón 2011}

Para determinar las concentraciones letales del aceite de trementina en esta cepa se expusieron larvas a cinco o más concentraciones del aceite de trementina disuelto en acetona, que causaron mor- talidades entre 2 y $98 \%$. Se utilizaron cuatro réplicas y un control por cada concentración. $\mathrm{La}$ mortalidad se determinó 24 horas después de aplicadas las diferentes dosis. Los resultados se analizaron mediante el programa Probit-logaritmo de Raymond (1985).

Se realizaron dos variantes, utilizando la $\mathrm{CL}_{95}$ obtenida para este aceite. En la variante I, se expusieron al aceite 1. 500 larvas en 10 recipientes con capacidad para $500 \mathrm{ml}$ de agua, a razón de 150 larvas en cada uno. A las 24 horas, se extrajeron las larvas muertas y las sobrevivientes se mantuvieron en el agua de exposición hasta la pupación. En la variante II, se procedió a exponer un igual número de larvas, con la modificación de que las sobrevivientes fueron trasladadas para agua limpia hasta el final de su ciclo biológico. En ambas variantes, las larvas fueron alimentadas con harina de pescado. Se contó diariamente la mortalidad de larvas y pupas. Las pupas sobrevivientes se sexaron y separaron hasta la eclosión de los adultos. Ambos experimentos se replicaron tres veces para un total de 4. 500 larvas utilizadas por cada variante. El tanto por ciento de inhibición de la emergencia (IE\%) se calculó utilizando la siguiente fórmula (WHO 2005):

$$
I E=\frac{100-T \times 100}{C}
$$

Donde $\mathrm{T}=$ tanto por ciento de sobrevivientes o adultos emergidos en los recipientes tratados.

$\mathrm{C}=$ tanto por ciento de sobrevivientes o adultos emergidos en los controles.

Los adultos sobrevivientes fueron colocados en una jaula de $30 \mathrm{~cm}$ x $30 \mathrm{~cm}$ x $30 \mathrm{~cm}$. Se les suministró alimentación con sangre. Se recogieron los huevos correspondientes al primer ciclo gonadotrófico, los cuales fueron puestos posteriormente a eclosionar, para medir la fecundidad como el total de huevos puestos y la fertilidad como huevos eclosionados.

Se utilizó un control correspondiente a la misma cepa San Miguel del Padrón 2011con 150 larvas en $500 \mathrm{ml}$ en cada experimento. Se sexaron las pupas y fueron colocadas en jaulas en similar proporción a los adultos obtenidos en cada variante. De igual manera se recogieron los huevos correspondientes al primer ciclo gonadotrófico, los cuales fueron puestos posteriormente a eclosionar para medir la fecundidad como el total de huevos 
puestos y fertilidad como huevos eclosionados.

En el análisis estadístico para comparar la mortalidad alcanzada entre las variante para cada estadio se realizó la prueba Mann Whitney. Se utilizó también un Anova Multifactorial para el análisis de la mortalidad diaria en todos los estadios de ambas variantes y para analizar la proporción de individuos por sexo obtenidos por variante. Se aplicó la prueba t de Student para comparar el número total de huevos puestos y el tanto por ciento de huevos eclosionados en ambas variantes.

\section{Resultados}

Determinación del estado de resistencia y /o susceptibilidad a insecticidas de la cepa colectada

Mediante los bioensayos de susceptibilidad y/o resistencia a los insecticidas adulticidas utilizados, se determinó que la cepa San Miguel del Padrón 2011 es resistente para Lambdacialotrina, Deltametrina, Malatión, Bendiocarb, al obtener una mortalidad de 76,3\%, 56,7 \%, 54,6 \% y 55,0 \%, respectivamente, a las 24 horas.

Al calcular los FR se encontró que los valores más altos correspondieron a los insecticidas Lambadacialotrina, Deltametrina y Malation, lo que concuerda con la resistencia detectada (Tabla 1). Sin embargo, frente a Bendiocarb la cepa en estudio fue susceptible $(\mathrm{FR}=3,5)$. Al evaluar la cepa al larvicida Temefos, se obtuvo un $40 \%$ de mortalidad, a la dosis diagnóstico, mientras que el FR tuvo un valor cercano a 5 , por lo que a nuestro criterio la cepa mostró una incipiente resistencia a este larvicida (Tabla 1).

\section{Obtención del aceite de trementina modificado}

El análisis reveló un elevado porcentaje de conversión de la mezcla monoterpénica mayoritaria primaria del aceite de trementina ( $\alpha / \beta$-pinenos, $>70 \%$ ), por el mecanismo carbocatión- $\alpha$ oxidación, en sistemas monocíclicos oxigenados tipo terpinolenos y limonenos, y derivados pulegona y verbenona. La generación de estos derivados, incluyendo la oxidación de los monociclos en posición alfa al doble enlace (pulegona-verbenona) e inclusión de un átomo de oxígeno en el sistema cíclico, se debe a la acción sinérgica de ácidos de Lewis tipo $\mathrm{V}_{2} \mathrm{O}_{5}, \mathrm{Nb}_{2} \mathrm{O}_{5}$ en presencia de oxígeno y foto-inducción (Stefani 1995, Tacoronte 2005).

\section{Evaluación de la actividad inhibidora del aceite de trementina modificado por foto- isomerización en larvas de Aedes aegypti resistente a insecticidas}

Al evaluar las diferentes dosis del aceite de trementina modificado se obtuvo la $\mathrm{CL}_{95}$, necesaria para lograr un efecto subletal. La cepa fue susceptible a este aceite pues se obtuvo un $\mathrm{FR}_{95}$ de 2,5, al compararla con la cepa Rockefeller (Tabla 2).

Por otra parte, se obtuvo un porcentaje de inhibición de $70 \%$, para la variante I y un $\mathrm{IE} \%=68 \%$ para la variante II. No se encontró diferencia significativa al comparar las mortalidades obtenidas en las fases larva, pupa y adulta entre de ambas variantes $(\mathrm{U}=195, p=0,52 ; \mathrm{U}=204, p=0,68 ; \mathrm{U}=185$ $p=0,37$ respectivamente) (Fig 1). Este resultado indica que el daño en las larvas sobrevivientes es el mismo; ya sean mantenidas en el agua de exposición hasta pupas, o si son expuestas solamente

\begin{tabular}{|c|c|c|c|}
\hline & $\begin{array}{l}\text { Rockefeller } \\
\text { TL }_{50}(\text { h }) /(\text { LC) }\end{array}$ & $\begin{array}{l}\text { San Miguel del Padrón } 2011 \\
\mathrm{TL}_{50}(\mathrm{~h}) /(\mathrm{LC})\end{array}$ & $\mathbf{F R}_{50}$ \\
\hline Lambadacialotrina & $0,29 /(0,25-0,32)$ & $2,87 /(2,42-3,63)$ & 9,8 \\
\hline Deltametrina & $0,34 /(0,32-0,36)$ & $2,23 /(1,93-2,67)$ & 6,5 \\
\hline Malation & $0,23 /(0,20-0,26)$ & $1,69 /(1,43-2,08)$ & 7,4 \\
\hline \multirow[t]{2}{*}{ Bendiocarb } & $0,52 /(0,50-0,54)$ & $1,87 /(1,62-2,21)$ & 3,5 \\
\hline & $\begin{array}{c}\text { Rockefeller } \mathrm{CL}_{50}(\mathrm{mg} / \mathrm{ml}) / \\
\text { (LC) }\end{array}$ & $\begin{array}{l}\text { San Miguel del Padrón } 2011 \\
\mathrm{CL}_{50}(\mathrm{mg} / \mathrm{ml}) /(\mathrm{LC})\end{array}$ & $\mathbf{F R}_{50}$ \\
\hline Temefos & $\begin{array}{c}0,00535 \\
(0,0054-00058)\end{array}$ & $\begin{array}{c}0,024 \\
(0,020-0,029)\end{array}$ & 4,4 \\
\hline
\end{tabular}

Tabla 1. Valores de concentración letal media $\left(\mathrm{TL}_{50}\right)$ y factor de resistencia $\left(\mathrm{FR}_{50}\right)$ de las cepas San Miguel del Padrón 2011 y Rockefeller frente a 4 insecticidas.

Table 1. Median lethal concentration values $\left(\mathrm{TL}_{50}\right)$ and resistance factors $\left(\mathrm{FR}_{50}\right)$ strains San Miguel del Padrón 2011 and Rockefeller against 4 insecticides. 


\begin{tabular}{|c|c|c|c|c|}
\hline CEPAS & $\begin{array}{c}\mathbf{C L}_{\mathbf{5 0}}(\mathbf{m g} / \mathbf{m l}) \\
(\mathbf{L C})\end{array}$ & $\begin{array}{c}\mathbf{C L}_{\mathbf{9 5}}(\mathbf{m g} / \mathbf{m l}) \\
(\mathbf{L C})\end{array}$ & FR $_{\mathbf{9 5}}$ & $\begin{array}{c}\mathbf{b} \\
\text { ( } \mathbf{D E})\end{array}$ \\
\hline Rockefeller & $\begin{array}{c}0,0023 \\
(0,0020-0,0026)\end{array}$ & $\begin{array}{c}0,0043 \\
(0,0041-0,0048)\end{array}$ & - & $6,32( \pm 0,66)$ \\
\hline San Miguel del Padrón 2011 & $\begin{array}{c}0,0058 \\
(0,0052-0,0060)\end{array}$ & $\begin{array}{c}0,011 \\
(0,0099-0,015)\end{array}$ & 2,5 & $5,37( \pm 0,87)$ \\
\hline
\end{tabular}

Tabla 2 Evaluación del aceite de trementina modificado en las cepas San Miguel del Padrón 2011 y Rockefeller.

Table 2. Evaluation of turpentine oil modified strains San Miguel del Padrón 2011 and Rockefeller.

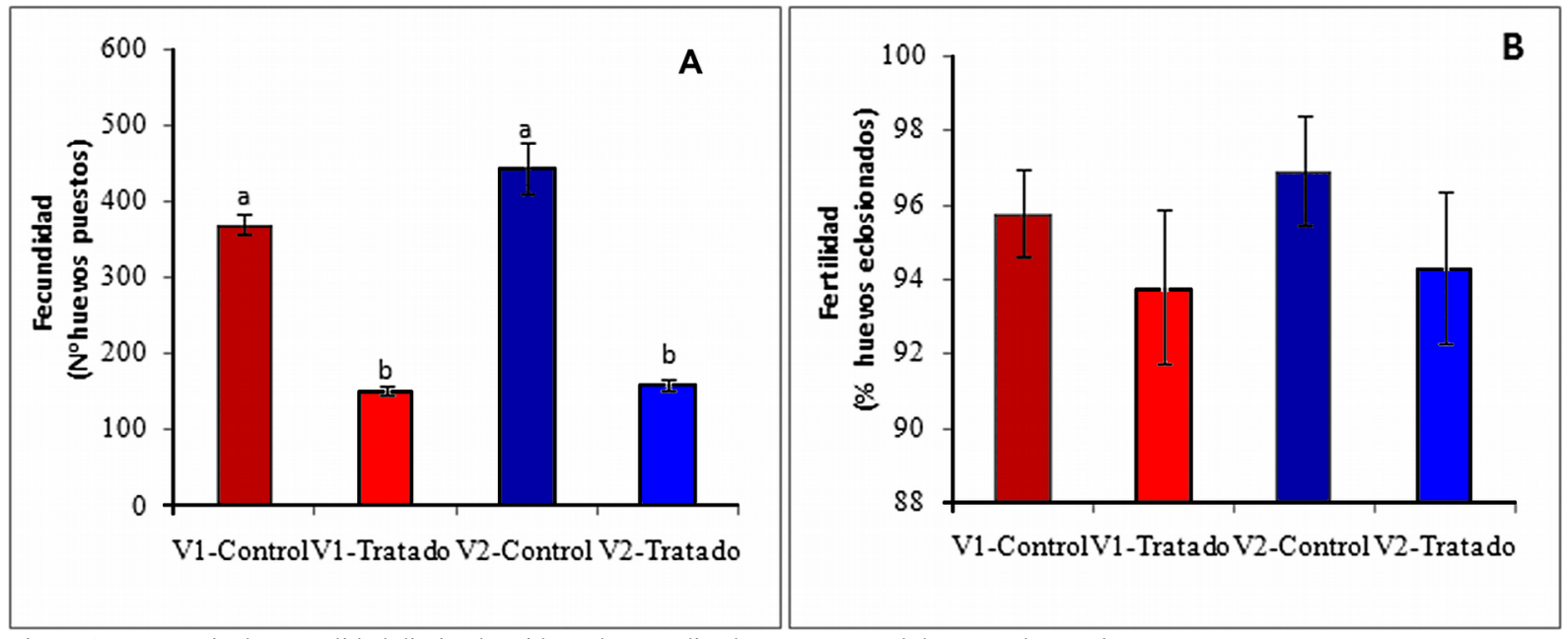

Figura 1. Porcentaje de mortalidad diaria obtenida en los estadios larva, pupa y adulto en ambas variantes.

Figure 1. Daily mortality percentage obtained in larva, pupa and adult in both variants.
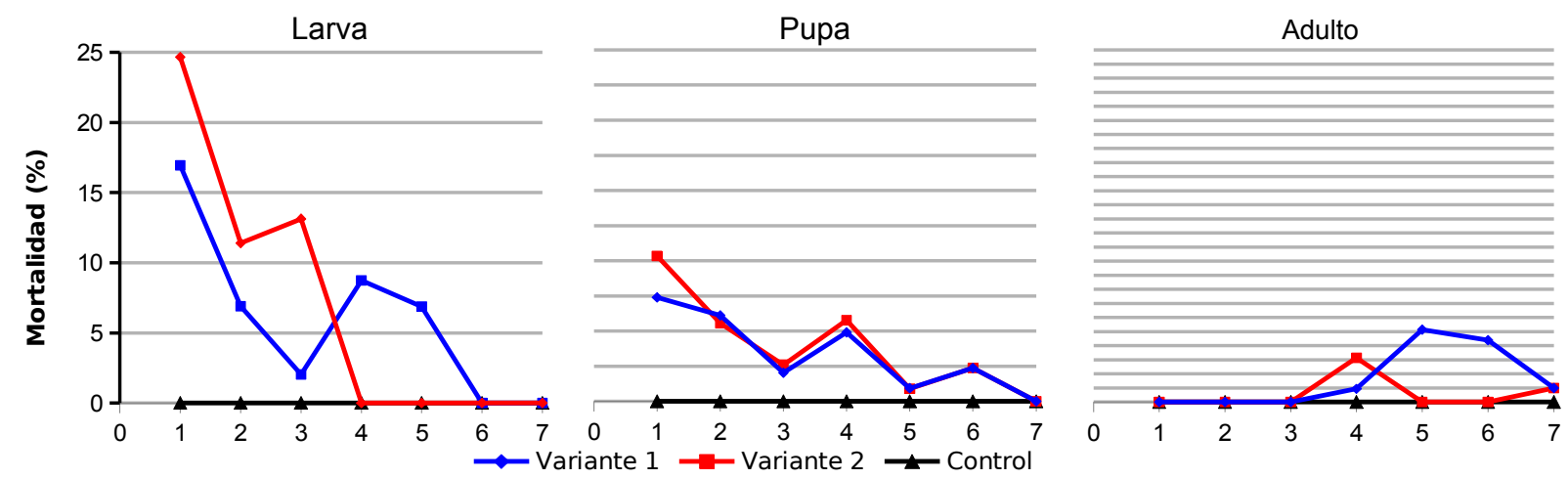

Figura 2. Efecto del aceite trementina sobre la fecundidad y fertilidad de $A$. aegypti en cada variante. (V1 y V2 son las variantes 1 y 2 respectivamente). Se realizó un ANOVA multivariado para ver las diferencias de las variables fecundidad y fertilidad para en cada grupo (control y tratado) de cada variante.

Figure 2. Effect of turpentine oil on fecundity and fertility of $A$. aegypti in each variant. (V1 and V2 are the variants 1 and 2 respectively). Multivariate ANOVA was conducted to see the differences in fecundity and fertility variables for each group (control and treated) of each variant.

por 24 horas. Sin embargo, si se encontró una variación entre el porciento de mortalidad en la fase larval con respecto a la fase pupal y a la fase adulta de cada variante $(\mathrm{F}=29,88, p=0,000001)$ (Fig. 1), lo que se asocia a que la cantidad de individuos disminuye respecto al valor inicial según transcurren los días del experimento. Se encontró diferencia significativa $(\mathrm{F}=18,77 p=0,003)$ entre el número de machos y hembras obtenidos en ambas variantes, lo que evidencia una afectación en la proporción sexual del número de individuos sobrevivientes para cada variante.

En cuanto al número de huevos puestos y huevos eclosionados, se encontró diferencia significativa para la fecundidad en ambas variantes con respecto al control $(\mathrm{T}=17,22, \quad p=0,000067$; 
$\mathrm{T}=3,54, p=0,024)$ (Fig 2A), no así para la fertilidad $(\mathrm{T}=1,90, p=0,129 ; \mathrm{T}=1,93, \mathrm{p}=0,125) \quad($ Fig 2B), lo que evidencia que existe afectación en la fisiología de los individuos sobrevivientes, dada por la disminución en el número de huevos puestos, independientemente de la variante usada.

\section{Discusión}

La presión selectiva con plaguicidas es capaz de sostener un incremento positivo de los individuos resistentes; mientras más presión se ejerza sobre el vector, más rápidamente se desarrolla el fenómeno de resistencia (Bisset 2002). La resistencia a Temefos se ha incrementado en la actualidad debido a su uso continuo en diferentes países (Chen et al. 2005, Marcombe et al. 2009, Melo-Santos 2010, Komalamisra et al. 2011, Cahyo et al. 2012). En Cuba existen trabajos sobre la pérdida de susceptibilidad a este larvicida (Rodríguez et al. 2004, Bisset et al. 2004). Los niveles de resistencia encontrados a Temefos, en la cepa San Miguel del Padrón 2011, son atribuibles a la presión de selección con este larvicida, aplicado durante más de 30 años en programa de control vectorial cubano. Con respecto al carbamato Bendiocarb, a pesar de no haber sido usado en Cuba para el control de Ae. aegypti, pensamos que el FR obtenido sea atribuible al uso del Ficam 80 WP para tratamiento perifocal en la provincia La Habana desde el año 2008. Sin embargo, Seau-Rong et al. (2012) si encontraron resistencia a este insecticida a pesar de no haber sido utilizado para el control vectorial en su país.

Una explicación complementaria para la resistencia encontrada a Malatión y Deltametrina, a pesar del que el primero se dejó de usar en la década de los 80 en nuestro programa de control y la Deltametrina se ha se aplicado en muy pocas localidades, es que la resistencia se haya alcanzado por el desarrollo de resistencia cruzada debido al uso de Temefos. Rodríguez et al. (2003) mostraron que la presión durante cinco generaciones con Malatión en larvas de Ae. aegypti de Cuba, no seleccionó una alta resistencia para ese insecticida pero si para piretroides como Deltametrina y Lambdacialotrina. De manera similar, Tikar et al. (2009) señalan que la selección de resistencia a Temefos en larvas puede generar resistencia cruzada a Permetrina en adultos. Si bien, en nuestro estudio, no es posible demostrar que el desarrollo de la tolerancia a Temefos haya generado resistencia cruzada con Lambdacialotrina, Deltametrina y Malatión, los resultados de Rodríguez et al. (2002, 2004, 2005, 2008) y Melo-Santos et al. (2010) apoyan la posibilidad de que esto ocurra.

Debido al surgimiento de resistencia a diferentes insecticidas en poblaciones naturales, en nuestro laboratorio se ha realizado la evaluación de aceites esenciales como una alternativa de control de Ae. aegypti. Al evaluar la cepa San Miguel del Padrón 2011 encontramos un ligero aumento de la $\mathrm{CL}_{95}$ para este aceite, cuando la comparamos con estudios previos realizados con la cepa Rockfeller. En ellos se determinó la actividad larvicida e inhibitoria de aceite de trementina (Leyva et al. 2010). Estudios realizados por Sutthanont et al. (2010), donde se evaluaron 5 aceites de plantas en una cepa resistente y en una susceptible a piretroides, se plantea que los aceites fueron ligeramente más tóxicos en la cepa susceptible que en la resistente. Pero, al igual que en nuestro estudio, no dan una respuesta definitiva de cual es la causa. Sin embargo, Tripathy et al. (2011), al analizar el efecto de 2 extractos de plantas sobre Culex quinquefasciatus Say, 1823 y Anopheles stephensi Liston, 1901, resistentes a insecticidas, encontraron un incremento de la actividad de glutation S-transferasa, no así en la cepa de Ae. aegypti. Somos del criterio que la resistencia metabólica a insecticidas, tanto para piretroides como organofosforados, que pudiera estar en la cepa San Miguel del Padrón 2011, pudiese ejercer influencia sobre la acción insecticidas de los aceites de plantas. En Cuba, no está extendido el uso de pesticidas de origen vegetal, por lo que la resistencia cruzada a los productos naturales es poco probable. Sin embargo, la causa del comportamiento de la resistencia en esta cepa pudiera estar relacionada con que el modo de acción del aceite de trementina modificado sea similar al de los insecticidas sintéticos. Por tanto, debemos hacer estudios posteriores donde se descarten los mecanismos de acción como glutatión S-transferasa, acetil colinesterasa y otras enzimas de acción metabólica.

Entre los plaguicidas se reconoce un grupo denominado reguladores del crecimiento, que tienen como principal acción actuar sobre las funciones metabólicas del insecto, alterando su metamorfosis. El desequilibrio de este sistema puede conllevar a la formación de cutículas anormales, no sólo en cuanto a su aspecto externo sino también en 
cuanto a su ultraestructura o nivel molecular, ya que se ha visto modificado su contenido proteico (Viñuela 1991). Los metabolitos secundarios presentes en las plantas le sirven como defensa frente a insectos, provocando repelencia, inhibición de la alimentación y/o actividad insecticida (Akhtar et al. 2003). Algunos de estos compuestos, conocidos como "disruptores endocrinos", son estructuralmente similares a hormonas. Diversos autores han demostrado la influencia de los metabolitos secundarios en el desequilibrio entre la ecdisona y hormona juvenil, alterando, por consiguiente, la metamorfosis del insecto. Esta variación genera mudas prematuras o tardías y la muerte de los individuos por no poder emerger de las pupas (Viñuela et al 1991, Agarwal et al. 2001, De Fur 2004, Céspedes et al. 2004, Burgueño-Tapia et al. 2008, Hincapié et al. 2011).

En nuestro estudio, por los resultados obtenidos, podemos inferir que el aceite de trementina modificado, se comporta como un regulador del crecimiento, primeramente provocando mortalidad larval y pupal y luego mortalidad de adultos, que quedan pegados a exuvias. También se afecta la proporción sexual y la puesta de huevos. En la variante II se obtienen mayor cantidad de hembras que machos en los adultos sobrevivientes. Este resultado puede estar relacionado con que, a las 48 horas de comenzado el experimento en esta variante, ocurrió la mayor mortalidad de pupas machos. El desarrollo biológico lento de las hembras respecto al de los machos trae como consecuencia que las pupas hembras se demoren más en emerger. En la variante I, no ocurre afectación en los sobrevivientes porque, al estar las larvas expuestas durante todo el tiempo, la mortalidad ocurre mayormente en larvas y, posteriormente, en pupas de ambos sexos. Resultados similares se han obtenido en trabajos realizados con derivados de la verbenona y pulegona, productos obtenidos de la modificación del aceite de trementina. Gunderson et al (1985) encontró que la administración diaria de pulegona en la dieta de Spodoptera eridania (Cramer, 1784) (Lepidoptera: Noctuidae) causó actividad anti-alimenticia logrando afectación en el peso, la fecundidad y viabilidad de sus huevos. En larvas de Ae. aegypti pulegona mostró actividad larvicida, repelente e inhibidora de la oviposición Waliwitiya et al (2009), mientras Fetting et al (2012), en un estudio con Pinus ponderosa Douglas, encontró actividad repelente de verbenona contra Dendroctonus brevicomis LeConte, 1876 (Coleoptera: Curculionidae).

En nuestro estudio, se demuestra que el aceite de trementina modificado actúa de la misma forma en una cepa susceptible (Leyva et al 2010) que en una resistente a insecticidas, provocando muerte larval y pupal en las larvas sobreviviente a la exposición. Además, afecta la proporción sexual y disminuye la fecundidad. Resultados análogos se encuentran con el pyriproxifeno y el diflubenzuron (inhibidores del desarrollo sintéticos), los que, además de su actividad larvicida típica, han causado disminución de la fertilidad y la fecundidad de los adultos provenientes de larvas sobrevivientes a la exposición a dosis subletales. Estos efectos se han encontrado en una gran variedad de insectos (Liu \& Chen 2001, Liu 2003, Mojaver \& Bandani 2010, Richardson \& Lagos 2007, Boina 2010, Navarro-Bueno 2007, Terán-Zavala 2012)

\section{Conclusiones}

El aceite de trementina modificado provocó alta mortalidad larval y pupal en las larvas de la cepa San Miguel del Padrón 2011 sobrevivientes de la exposición con la $\mathrm{CL}_{95}$. Se obtuvo para la variante I un $70 \%$ de inhibición y para la variante II un 68 $\%$. Las hembras sobrevivientes de ambas variantes disminuyeron su fecundidad con respecto al control. Los resultados logrados evidencian el efecto inhibidor del desarrollo del aceite.

\section{Referencias}

Agarwal M, Walia S, Dhingra S \& Khambay BP. 2001. Insect growth inhibition, antifeedant and antifungal activity of compounds isolated/derived from Zingiber officinale Roscoe (ginger) rhizomes. Pest Management Science 57(3): 289-300.

Akhtar Y, Rankim CH Isman MB. 2003. Decreased response to feeding deterrents following prolonged exposure in the larvae of a generalist herbivore, Trichoplusia ni (Lepidoptera: Noctuidae). Journal of Insect Behavior 16(6): 811-831.

Barra A, Coroneo V, Dessi S, Cabras P \& Angioni A. 2007. Characterization of the volatile constituents in the essential oil of Pistacia lentiscus $L$ from differents origins and its antifungal and antioxidant activity. Journal of Agricultural Food Chemical 55(17): 7093-7098.

Bisset JA. 2002. Uso Correcto de Insecticidas: Control de la Resistencia. Revista Cubana Medicina Tropical. 54(3): 202-219.

Bisset JA, Rodríguez MM, Fernández D \& Pérez $O$. 
2004. Estado de la resistencia en larvas del municipio Playa colectadas durante la etapa intensiva contra el Aedes aegypti en Ciudad de la Habana 20012002. Revista Cubana Medicina Tropical 56(1): 6166.

Boina D, Rogers M, Wang N \& Stelinski L. 2010. Effect of pyriproxyfen, a juvenile hormone mimic, on egg hatch, nymph development, adult emergence and reproduction of the Asian citrus psyllid, Diaphorina citri Kuwayama. Pest Management Science 66(4): 349-357.

Bourguet D, Genissel A \& Raymond M. 2000. Insecticide resistance and dominance levels. Journal of Economic Entomology 93(6): 1588-1595.

Bouvier JC, Bues R, Boivin LB, Beslay D\& Sauphanor B. 2001. Deltamethrin resistance in the codling moth (Lepidopthera: Tortricedae): inheritance and number of genes involved. Heredity 87: 456-462.

Burgueño-Tapia E, Castillo L, González-Coloma A \& Joseph-Nathan P. 2008. Antifeedant and phytotoxic activity of the sesquiterpene p-benzoquinone perezone and some of its derivatives. Journal Chemical Ecology. 34(6): 766-771.

Brown AWA. 1986. Insecticide resistance in mosquitoes: pragmatic review. Journal American Mosquito Control Association 2: 123-140.

Cantin A, Lull C, Prima J, Miranda MA \& Primo-Yuñera E. 2001. Isolation structural assignment and insecticdal activity of (-)-(1S,2R,3R,4S)-1,2-epoxy-1metyl-4-(1-methyllethyl)-cyclohex-3-yl acetate a natural product from Minthostachys tormentosa. Tetrahedron: Asymmetry 87: 677-683.

Cahyo Mulyatno K, Yamanaka A, Konishi N \& Konishi N. 2012. Resistance of Aedes aegypti (L) larvae to temephos in Surabaya, Indonesia. Southeast Asian Journal Tropical Medical Public Health 43(1): 29-33.

Céspedes CL, Torres P, Marín JC, Arciniegas A, Romo de Vivar A, Pérez-Castorena AL Aranda E. 2004. Insect growth inhibition by tocotrienols and hydroquinones from Roldana barba-johannis. Phytochemistry 65(13): 1963-1975.

Chen CD, Nazni, WA, Lee HL \& Sofian-Azirun M. 2005. Weekly variation on susceptibility status of Aedes mosquitoes against temephos in Selangor, Malaysia. Tropical Biomedicine 22(2): 195-206.

Conti B, Canale A, Bertolli A, Gozzini F\& Pistelli L. 2010. Essential oil composition and larvicidal activity of six meditarranean aromatic plants agianst the mosquito Aedes albopictus (Diptera: Culicidae). Parasitolgy Research 107(6): 1455-1461.

Dharmagadda VSS, Naik SN, Mittal PK \& Vasudevan P. 2005. Larvicidal activity of Tagetes patula essential oil against three mosquito species. Bioresource Technology 96: 1235-1240.

Defur PL. 2004. Use and role of invertebrate models in endocrine disruptor research and testing. Institute for Laboratory Animal Research Journal 45(4): 484493.

Erturk O. 2006. Antifeed and toxicity effects of some plants extracts on Thaumetopae solitaria Frey (Lep.: Thaumetopoeidae). Turkish Journal of Biology 30(1): 51-57.
Fettig CJ, McKelvey SR, Dabney CP, Huber PW, Lait CG, Fowler DL \& Borden JH. 2012. Efficacy of "Verbenone Plus" for Protecting Ponderosa Pine Trees and Stands from Dendroctonus brevicomis (Coleoptera: Curculionidae) Attack in British Columbia and California. Journal of Economic Entomology 105(5): 1668-1680.

Garcia M, Dona del OJ, Ardanaz CE, Tonn CE Sosa ME. 2005. Toxic and repellent effects of Baccharis salicifolia essential oil on Tribulium castaneum. Pest Management Science 61(6): 612-618.

Gunderson CA, Samuelian JH! Evans CK. 1985. Effects of the mint monoterpene pulegone on Spodoptera eridania (Lepidoptera: Noctuidae). Environmental Entomology 14(6): 859-863.

Hincapié CA, Monsalve Z, Parada K, Lamilla C, Alarcón J, Céspedes CL \& Seigler D. 2011. Insect growth regulatory activity of Blechnum chilense. Natural Product Communication 6(8): 1085-1058.

Kirk R E, Othemer DF. 1983. Turpentine. Encyclopedia of chemical technology 14: 381-97

Keiding J. 1986. Prediction or resistance Risk Asseaament. National Academy Press, Washington. DC. pp. 279- 297.

Komalamisra N, Srisawat R, Phanbhunwong T\& Oatwaree S. 2011. Insecticide susceptibility of the Dengue vector Aedes aegypti (L) in metropolitan Bangkok. Southeast Asian Journal Tropical Medical Public Health. 42 (4): 814-823.

Leyva M, Tacoronte JE, Marquetti MC, Scull R, Tiomno O, Mesa A \& Montada D. 2009. Utilización de aceites esenciales de pináceas endémicas como una alternativa de control en Aedes Aegypti Revista Cubana Medicina Tropical 61(3): 239-43

Leyva M, Marquetti MC, Tacoronte JA, Tiomno O \& Montada D. 2010. Efecto inhibidor del aceite de trementina sobre el desarrollo de larvas de Aedes aegypti (Diptera: Culicidae) Revista Cubana Medicina Tropical 62(3): 212-216.

Leyva M, Castex M, Montada D, Quintana F, Lezcano D, Marquetti MC, Companioni A, Anaya J\& González I. 2012. Actividad repelente de formulaciones del aceite esencial de Melaleuca quinquenervia (Cav.) S. T. Blake (Myrtales: Myrtaceae) en mosquitos. Anales de Biología 34: 47-56.

Liu TX \& Chen TY. 2001. Effects of a juvenile hormone analog pyriproxyfen on apterous from of Lipaphi serysimi (Homoptera: Aphididae). Entomologia Experimentalis et Applicata 98: 295-301.

Liu TX. 2003. Effects of a juvenile hormone analog pyriproxyfen on Thrips tabaci (Thysanoptera: Thripidae) Pest Management Science 59(8): 904-912.

Llinas GA, Seccacini E, Gardenal CN \& Licastro S. 2010. Current resistance status to temephos in Aedes aegypti from different regions of Argentina. Memorias do Instituto Oswaldo Cruz 105: 113-116.

Marcombe S, Poupardin R, Darriet F, Reynaud S, Bonnet J, Strode C, Brengues C, Yebakima A, Ranson $\mathrm{H}$, Corbel V \& David JP. 2009. Exploring the molecular basis of insecticide resistance in the dengue vector Aedes aegypti: a case study in Martinique Island (French West Indies). BMC Genomics 10: 494. 
Melo-Santos MAV, Varjal-Melo JJM, Araújo AP, Gomesa TCS, Paivaa MHS, Regis LN, Furtado AF, Magalhaes T, Macoris MLG, Andrighetti MTM \& Ayres CFJ. 2010. Resistance to the organophosphate temephos: Mechanisms, evolution and reversion in an Aedes aegypti laboratory strain from Brazil. Acta Tropica 113: 180-189.

Mojaver M. Bandani AR. 2010. Effects of the insect growth regulator pyriproxyfen on immature stages of Sunn Pest, Eurygaster integriceps Puton (Heteroptera: Scutelleridae). Munis Entomology \& Zoology 5(1): 187-197.

Montella IR, Martins AJ, Viana-Medeiros PF, Lima JB, Braga IA \& Valle D. 2007. Insecticide resistance mechanisms of Brazilian Aedes aegypti populations from 2001 to 2004. The American journal of tropical medicine and hygiene 77: 467-477.

Navarro-Bueno E, Berti-Moser J \& González-Rivas J. 2007. Efecto de metopreno sobre la fecundidad y fertilidad de hembras de Anopheles albimanus expuestas en fase de larva a una concentración subletal del producto. Boletín de Malariologia y Salud Ambiental 47(2): 249-251.

OMS. Instrucciones para determinar la susceptibilidad o resistencia a insecticidas en larvas de mosquito. WHO/VBC/81. 807.

Pascual-Villalobos MJ \& Ballesta-Acosta MC. 2003. Chemical variation in an Ocimun basilicum germplasm collection and activity of the essential oils on Callosobruchus maculatus. Biochemical Systematics and Ecology 31(7): 673-679.

Pascual-Villalobos MJ. 1998. Repelencia, inhibición del crecimiento y toxicidad de extractos vegetales en larvas de Tribolium castaneum Herbst (Coleoptera: Tenebririonidae) Boletín de Sanidad Vegetal. Plagas 24(1): 143-154.

Pérez O, Rodríguez J, Bisset JA, Leyva M, Díaz M, Fuentes O, Ramos F, González R \& García I. 2004. Manual de Indicaciones Técnicas para Insectarios. Editorial Ciencias Medicas, Cuba pp. 59

Raymod M. 1985. Presentation d'un prograamme d'analyce log probit pacer micro-ordenateur. Cah Cahiers. Cahiers O.R.S.T.O.M. Série entomologie médicale et parasitologie 22: 117-121.

Richardson ML \& Lagos DM. 2007. Effects of a juvenile hormone analogue, pyriproxyfen, on the apterous form of soybean aphid (Aphis glycines). Journal of Applied Entomology 131(5): 297-302.

Rodriguez M, Bisset JA Ruiz M \& Soca A. 2002. Crossresistance to pyrethroid and organophosphorus insecticides induced by selection with temephos in Aedes aegypti (Diptera: Culicidae) from Cuba. Journal of Medical Entomology 39: 882-888.

Rodríguez M,Bisset JA, Diaz C \& Soca A. 2003. Resistencia cruzada a piretroides en Aedes aegypti de Cuba inducido por la selección con el insecticida organofosforado malatión. Revista Cubana de Medicina Tropical 55 (2): 105-111.

Rodriguez MM, Bisset JA, Fernández D \& Pérez $O$. 2004. Resistencia a insecticidas en larvas y adultos de Aedes aegypti: prevalencia de la esterasa A4 asociada con la resistencia a temefos. Revista $\mathrm{Cu}$ - bana Medicina Tropical 56(1): 54-60.

Rodriguez M, Bisset JA, De Armas Y \& Ramos F. 2005. Pyrethroid insecticide-resistant strain of Aedes aegypti from Cuba induced by Deltamethrin selection. Journal of the American Mosquito Control Association. 21 (4): 437-445.

Rodriguez M. 2008. Estudio de la resistencia a insecticidas. Tesis doctoral. Instituto de Medicina Tropical Pedro Kouri $114 \mathrm{p}$.

Rodríguez MM, Bisset JA, Pérez O, Montada D, Moya M, Ricardo $Y$ \& Valdés V. 2009. Estado de la resistencia a insecticidas y sus mecanismos en Aedes aegypti en el municipio Boyeros. Revista Cubana Medicina Tropical 61(2):

Rodríguez MM, Bisset JA, RicardoY, Pérez O, Montada D, Figueredo D \& Fuentes D. 2010. Resistencia a insecticidas organofosforados en Aedes aegypti (Diptera: Culicidae) de Santiago de Cuba, 1997-2009. Revista Cubana Medicina Tropical 62(3): 217-23

Shaalan EA, Canyon D, Younes MW, Abdel-Wahab H \& Mansour AH. 2005. A review of botanical phytochemicals with mosquitocidal potential. Environment Internacional 31(8): 1149-1166.

Silva G. Insecticidas vegetales. 2002. En: http: //ipmworld. umn. edu/ cancelado /Spchapters/GSilvaSp. $\mathrm{Htm}(11-12-2006)^{\star *}$

Seau-Rong L, Tan-Wei-Ann A, Wasi-Ahmad N, Ham-Lim L\& Sofian-Azirum M. 2012. Insecticidal susceptibility status of field collected Aedes (Stegomya) aegypti $(L)$ at dengue endemic site in Sham Alam Selengor Malasya. Southeast Asian Journal Tropical Medical Public Health 43(1): 34-47.

Sutthanont N, Choochote W, Tuetun B, Junkum A, Jitpakdi A, ChaithongU, Riyong D \& Pitasawat B. 2010. Chemical composition and larvicidal activity of edible plant-derived essential oils against the pyrethroidsusceptible and resistant strains of Aedes aegypti (Diptera: Culicidae). Journal of Vector Ecology 35(1): 106-115.

Stefanis A. 1995. Terpene conversions in presence of alumina -pillared clays and phosphates. Applied Catalysis A: General, 132, 335-365

Tacoronte JE 2005. Procedimiento catalítico de acetilación de alcoholes secundarios esteroidales y monoterpénicos Pat. CU Boletín Oficial OCPI. Resolución 2614/2005.

Terán Zavala María del Carmen. 2012. Niveles de Susceptibilidad a Temefos y Eficacia de Pyriproxifeno para el control de Aedes aegypti(Diptera: Culicidae)en la ciudad de Guayaquil - Ecuador. Tesis para optar por el grado de Master en Entomología Medica y Control de Vectores. Instituto Pedro Kourí. Cuba.

Tikar SN, Kumar A, Prasad GB \& Prakash S. 2009. Temephos-induced resistance in Aedes aegypti and its cross-resistance studies to certain insecticides from India. Parasitol Research 105(1): 57-63.

Tripathy A, Samanta L, Sachidananda D, Parida S, Marai n, Hazra RK, Mallavdani UV, Kar SK \&Mahapatra N. 2011. The mosquitocidal activity of methanolic extracts of Lantana camara Root and anacardium occidentalle leaf: role of GST in insecticidal resis- 
tance. Journal Medical Entomology 48(2): 291-295.

Viñuela E, Budin F. de Estal P. 1991. Los insecticidas reguladores del crecimiento y la cutícula. Boletín Sanidad Vegetal Plagas 17(3): 391-400.

Waliwitiya R, Kennedy CJ. Lowenberg CA. 2009. Larvicidal and oviposition alterin activity of monoterpenoids, trans anithole and rosmary oil to the yellow fever mosquito Aedes aegypti (Diptera: Culicidae) Pest Management Science 65(3): 241-248.

WHO/CDC/CPC/MAL. 98. 12 Report of the WHO Infor- mal Consultation. Test procedures for insecticide resistance, monitoring in malaria vectors. Bio efficacy and persistence of insecticides on treated surfaces.

WHO/VBC. 81. 806. 1981. Instructions for determining the susceptibility or resistance of adult mosquitoes to organochlorine, organophosphate and carbamates insecticides. Diagnostic test.

WHO/CDS/WHOPES/GCDPP/2005. 13 Guidelines for laboratory and field mosquito larvicida. Geneva. 2005 